\title{
Coulomb tunneling for fusion reactions in dense matter: Path integral Monte Carlo versus mean field
}

\author{
A. I. Chugunov \\ Ioffe Physico-Technical Institute, Politekhnicheskaya 26, 194021 Saint-Petersburg, Russia \\ H. E. DeWitt \\ Lawrence Livermore National Laboratory, Livermore, CA 94550, USA \\ D. G. Yakovlev \\ Ioffe Physico-Technical Institute, Politekhnicheskaya 26, 194021 Saint-Petersburg, Russia
}

\begin{abstract}
We compare Path Integral Monte Carlo calculations by Militzer and Pollock (Phys. Rev. B 71, 134303, 2005) of Coulomb tunneling in nuclear reactions in dense matter to semiclassical calculations assuming WKB Coulomb barrier penetration through the radial mean-field potential. We find a very good agreement of two approaches at temperatures higher than $\sim \frac{1}{5}$ of the ion plasma temperature. We obtain a simple parameterization of the mean field potential and of the respective reaction rates. We analyze Gamow-peak energies of reacting ions in various reaction regimes and discuss theoretical uncertainties of nuclear reaction rates taking carbon burning in dense stellar matter as an example.
\end{abstract}

PACS numbers:

\section{INTRODUCTION}

Nuclear fusion reactions in dense stellar matter affect the evolution of ordinary stars and compact stars such as white dwarfs and neutron stars. Hydrogen and helium burning, and later the burning of carbon and heavier elements [1] drives an ordinary star through the main sequence and giant/red-giant branch towards its final moments as a normal star. Explosive burning of carbon and other elements in the cores of massive white dwarfs triggers type Ia supernova explosions (see, e.g., [2] and references therein). Thermonuclear burning of accreted matter in surface layers of neutron stars, which enter compact binaries, produces type I X-ray bursts [3]. Deeper burning of carbon in accreting neutron stars is likely responsible for superbursts observed from some X-ray bursters (e.g., Refs. [4, 5] ). Even deeper burning of accreted matter in pycnonuclear reactions in the crust of transiently accreting neutron stars can power thermal radiation observed from neutron stars in soft X-ray transients in quiescent states (see, e.g., Refs. [5, 6, 7])). All in all, nuclear fusion is important in all stars at all evolutionary stages.

It is well known that nuclear reaction rates in dense matter are determined by astrophysical $S$-factors, which characterize nuclear interaction of fusing atomic nuclei, and by Coulomb barrier penetration preceding the nuclear interaction. We will mostly focus on the Coulomb barrier penetration problem. Fusion reactions in ordinary stars proceed in the so called classical thermonuclear regime in which ions (atomic nuclei) constitute nearly ideal Boltzmann gas. In this case the Coulomb barrier between reacting nuclei is almost unaffected by plasma screening effects produced by neighboring plasma particles. The Coulomb barrier penetrability is then well defined.

However, in dense matter of white dwarf cores and neutron star envelopes the ions form a strongly non-ideal Coulomb plasma, where the plasma screening effects are very strong. Plasma screening greatly influences the barrier penetrability and the reaction rates. Depending on the density and temperature of the matter, nuclear burning can proceed in four other regimes [8]. They are the thermonuclear regime with strong plasma screening, the intermediate thermo-pycnonuclear regime, the thermally enhanced pycnonuclear regime, and the pycnonuclear zero-temperature regime. The reaction regimes will be briefly discussed in Sec. III In these four regimes the calculation of the Coulomb barrier penetration is a complicated problem. There have been many attempts to solve this problem using several techniques but the exact solution is still a subject of debates. Various techniques and approaches will also be outlined in Sec. III.

In Sec. III we analyze recent Path Integral Monte Carlo (PIMC) calculations of fusion reaction rates by Militzer and Pollock [9]. We compare these results with those obtained within a much simpler formalism of semi-classical Coulomb tunneling in a mean-field potential. To this aim, in Sec. [V] we analyze and parameterize the mean field potential in a strongly non-ideal classical ion plasma and also calculate and parameterize mean-field reaction rates. Section $\nabla$ is devoted to comparison of calculations by different authors. We conclude in Sec. VI.

\section{NUCLEAR REACTION REGIMES}

\section{A. Physical parameters}

We consider a fusion reaction between identical nuclei $(A, Z)+(A, Z)$ in a one-component plasma (OCP) of atomic nuclei (ions) in dense matter. Here, $A$ and $Z$ are the mass and charge numbers of the nuclei, respectively. 


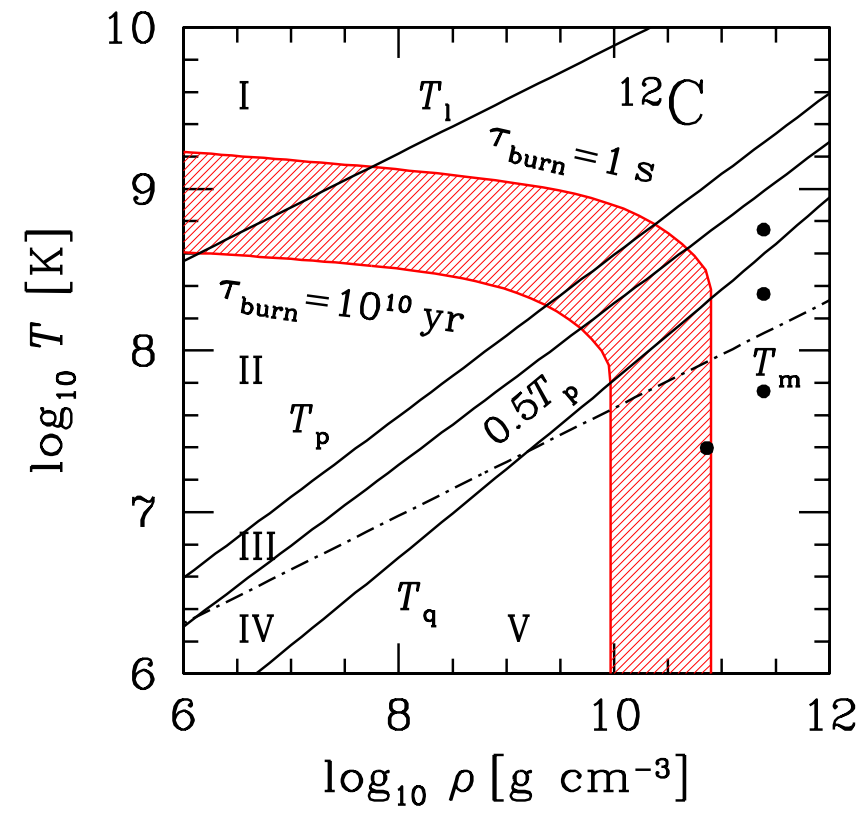

FIG. 1: (Color online) Temperature-density diagram for carbon matter; $T_{l}(\Gamma=1)$ is the temperature below which ions form strongly coupled liquid; $T_{p}(\zeta \approx 0.513)$ is the ion plasma temperature; $T_{m}$ is the solidification temperature in a classical ion liquid; $T_{q}$ is the temperature below which the carbon burning rate is temperature independent; I-V label domains of different nuclear burning regimes (Table 【). The shaded region is most important for applications of carbon burning; it is restricted by the lines of constant burning times ( $1 \mathrm{~s}$ and $10^{10} \mathrm{yr}$, for the upper and lower lines, respectively, from Ref. [10]). Filled dots show some $T-\rho$ points for which PIMC calculations [9] of reaction rates have been performed if applied to carbon burning.

The results will be general but they will be illustrated taking the ${ }^{12} \mathrm{C}+{ }^{12} \mathrm{C}$ reaction as an example that is most important for astrophysical implications (Sec. I). The temperature-density $(T-\rho)$ diagram for carbon matter is shown in Fig. 1. The filled dots show some $T-\rho$ points for which PIMC calculations [9] have been performed if applied to carbon burning. Notice that the majority of the data points [9] correspond to much higher $T$ and $\rho$, where carbon would be immediately transformed into other elements either through beta captures or through intense nuclear reactions. All the PIMC data [9] are analyzed in Secs. III V The shaded region in Fig. 11is briefly described in Sec. IIG.

Under the conditions displayed in Fig. 1] carbon is fully ionized and immersed in a nearly uniform electron background; the electrons are mostly strongly degenerate.

Coulomb coupling of ions is determined by the familiar parameter

$$
\Gamma=Z^{2} e^{2} /\left(k_{B} a T\right)
$$

where $a=\left[3 /\left(4 \pi n_{i}\right)\right]^{1 / 3}$ is the ion-sphere radius, $n_{i}$ is the ion number density, and $k_{B}$ the Boltzmann constant. At
TABLE I: Nuclear reaction regimes in dense matter [8].

\begin{tabular}{clc}
\hline Line & \multicolumn{1}{c}{ Regime } & Domain \\
\hline I & Thermonuclear with weak screening & $T \gg T_{l}$ \\
II & Thermonuclear with strong screening & $T_{p} \lesssim T \lesssim T_{l}$ \\
III & Thermo-pycnonuclear & $0.5 T_{p} \lesssim T \lesssim T_{p}$ \\
IV & Thermally enhanced pycnonuclear & $T_{q} \lesssim T \lesssim 0.5 T_{p}$ \\
V & $T=0$ pycnonuclear & $T \lesssim T_{q}$ \\
\hline
\end{tabular}

temperatures $T \gg T_{l}=Z^{2} e^{2} /\left(k_{B} a\right)$ (i.e., at $\left.\Gamma \ll 1\right)$ the ions constitute an almost ideal Boltzmann gas. At $T \lesssim T_{l}$ ( $\Gamma \gtrsim 1)$ they smoothly (without any phase transition) transform into a strongly coupled liquid. The ions solidify at $T=T_{m}$ which is much lower than $T_{l}$. The freezing into the classical body-centered cubic (bcc) crystal occurs at $\Gamma_{m} \approx 175$, that is at $T_{m}=T_{l} / \Gamma_{m}$. The difference of free energies of various Coulomb structures at low temperatures is very small, and the actual microstructure at these temperatures is rather uncertain (as discussed, e.g., in $[10,11])$.

The importance of quantum effects in motion of plasma ions can be characterized by the ion plasma temperature $T_{p}$ determined by the ion plasma frequency $\omega_{p}$,

$$
T_{p}=\hbar \omega_{p} / k_{B}, \quad \omega_{p}=\sqrt{4 \pi Z^{2} e^{2} n_{i} / m_{i}},
$$

where $m_{i}$ is the ion mass. At $T \gtrsim T_{p}$ quantum effects are relatively weak and quantization of ion motion is mainly unimportant. At lower $T$ quantum effects become most essential. At $\rho \gtrsim 4 \times 10^{11} \mathrm{~g} \mathrm{~cm}^{-3}$ dense matter contains free neutrons dripped off atomic nuclei.

OCP of ions can also be characterized by the parameters

$$
r_{s}=\frac{a}{a_{B}}, \quad \eta=\frac{\Gamma}{r_{s}}, \quad \zeta=\left(\frac{4 \Gamma^{2}}{\pi^{2} r_{s}}\right)^{1 / 3}=\left(\frac{4 T_{p}^{2}}{3 \pi^{2} T^{2}}\right)^{1 / 3}
$$

where $a_{B}=\hbar^{2} /\left(Z^{2} e^{2} m_{i}\right)$ is the ion Bohr radius. A state of ions is determined by two parameters, for instance, by $\Gamma$ and $T / T_{p}$ (or $\zeta$ ), while other parameters can be expressed through these ones.

We will also need a temperature (Fig. (1)

$$
T_{q}=0.5 T_{p} / \ln \left(T_{l} / T_{p}\right)
$$

which is the upper temperature of temperatureindependent pycnonuclear burning (Sec. IID).

The five nuclear reaction regimes [8] are summarized in Table [. Temperature-density domains for carbon burning in these regimes are seen from Fig. 1] We outline these regimes for fusion reactions of identical nuclei in an OCP. 


\section{B. Thermonuclear burning with weak plasma screening}

This regime (regime I in Table I) is realized at $T \gg$ $T_{l}$. In this case Coulomb coupling of ions is weak and Coulomb tunneling is only slightly affected by plasma screening effects. The main contribution to reaction rate comes from a small amount of ions with energies $E$ near the Gamow-peak energy $E_{\mathrm{pk}}$ (much higher than $k_{B} T$ ).

Neglecting the plasma screening effects, one obtains the familiar thermonuclear reaction rate

$$
R_{\mathrm{th}}=4 \frac{n_{i}^{2}}{2} \sqrt{\frac{2 E_{\mathrm{pk}}}{3 \mu}} \frac{S\left(E_{\mathrm{pk}}\right)}{k_{B} T} \exp (-\tau),
$$

where $E_{\mathrm{pk}}=k_{B} T \tau / 3, \mu=m_{i} / 2$ is the reduced mass, $S(E)$ is the astrophysical factor (assumed to be a slowly varying function of $E$ ), and

$$
\tau=\left(\frac{27 \pi^{2} \mu Z^{4} e^{4}}{2 k_{B} T \hbar^{2}}\right)^{1 / 3} .
$$

The plasma screening enhances the reaction rates. In the thermonuclear regimes with weak and strong screening (at $T \gtrsim T_{p}$, and actually even at somewhat lower $T$ ) the "screened" rate can be conveniently written as

$$
R_{\mathrm{th}}^{\mathrm{scr}}=R_{\mathrm{th}} F_{\mathrm{scr}}, \quad F_{\mathrm{scr}}=\exp (h),
$$

where $R_{\mathrm{th}}$ is given by Eq. (5) and $F_{\text {scr }}$ is the enhancement factor expressed through a function $h$. In the weak screening Debye-Hückel limit $\left(\Gamma \ll 1, T \gg T_{l}\right)$, one obtains [12] $h=\sqrt{3} \Gamma^{3 / 2} \ll 1$, so that $F_{\text {scr }} \approx 1+h$ is only slightly higher than 1 .

\section{Thermonuclear burning with strong plasma screening}

This regime (regime II in Table I) occurs at $T_{p} \lesssim T \lesssim$ $T_{l}$. The majority of ions are strongly coupled by Coulomb forces in their potential wells; quantum effects in their motion are weak. The main contribution to the reaction rate comes from a small amount of highly energetic ions which are nearly free and have Gamow-peak energies (modified by the screening effects). The plasma screening strongly enhances the reaction rate.

The screening is often modeled assuming that the reacting nuclei move in a potential

$$
U(r)=Z^{2} e^{2} / r-H(r),
$$

where $H(r)$ is a static and spherically symmetric meanfield plasma potential. This approach neglects fluctuations of plasma screening microfields during an individual tunneling event.

Generally, the function $h$ can be split into two parts,

$$
h=h_{0}+h_{1}, \quad F_{\text {scr }}=\exp \left(h_{0}\right) \exp \left(h_{1}\right) .
$$

The leading term $h_{0}=H(0) / k_{B} T$ is calculated assuming a constant mean-field plasma potential $H(r)=H(0)$ during the quantum tunneling, while $h_{1}$ is a correction owing to a weak variation of $H(r)$ along the tunneling path and owing to possible deviations from the mean-field approximation (concerned with fluctuations). Estimates show (e.g., Ref. [13]) that typical tunneling lengths of the reacting ions in the thermonuclear regime $\left(T \gtrsim T_{p}\right)$ are smaller than the ion sphere radius $a$, and typical tunneling times are shorter than the plasma oscillation time scales $\sim \omega_{p}^{-1}$. This justifies the approach of almost constant and static plasma potential during a tunneling event as a first approximation.

The main screening quantity $h_{0}$ is determined by $H(0)$. For a classical ion system, $H(0)$ can be calculated as $H(0)=\Delta \mathcal{F}$, where $\Delta \mathcal{F}$ is a difference of the Coulomb free energy for a given system of nuclei and for a system with two nuclei merged into one compound nucleus (e.g., DeWitt et al. [14]). In this case the leading enhancement factor $\exp \left(h_{0}\right)=\exp \left(\Delta \mathcal{F} / k_{B} T\right)$ depends on the one argument $\Gamma$.

The mean-field potential $H(r)$ for a classical strongly coupled OCP of ions (liquid or solid) can be determined from classical Monte Carlo (MC) sampling (e.g., DeWitt et al. [14]). We will analyze the latest results in Sec. IVA MC sampling gives the static classical radial pair distribution function of ions $g(r)$ which equals $g(r)=\exp \left[-U(r) / k_{B} T\right]$. In this way one obtains accurate values of $g(r)$ and $H(r)=Z^{2} e^{2} / r+k_{B} T \ln g(r)$ at not too small $r$ (typically, at $r \gtrsim a$ ), because MC statistics of close ion separations $r \lesssim a$ is poor due to strong Coulomb repulsion of ions at small distances. The potential $H(r)$ at small $r$, required for calculating the tunneling probability, can be obtained by extrapolating $\mathrm{MC}$ values of $H(r)$ to $r \rightarrow 0$. The extrapolation is a delicate procedure (as shown, e.g., by Rosenfeld [15]).

Assuming a linear mixing rule in a multi-component strongly coupled ion plasma, Jancovici [16] obtained

$$
h_{0}(\Gamma)=2 f_{0}(\Gamma)-f_{0}\left(2^{5 / 3} \Gamma\right),
$$

where $f_{0}(\Gamma)$ is a Coulomb free energy per one ion in an OCP (in units of $k_{B} T$ ). Using MC data available by that time (1977) he got $h_{0}(\Gamma)$ given in line (a) of Table! The same expression was used by Itoh et al. [19].

Recent MC calculations for a classical Coulomb liquid at $\Gamma \gtrsim 1$ give highly accurate values of $f_{0}(\Gamma)$ (accurately approximated by analytical functions, e.g., Ref. [20]) and confirm the validity of the linear mixing rule (e.g., Ref. 21]). The function $h_{0}(\Gamma)$ has been calculated from Eq. (10) in many papers (e.g., [13, 15, 16, 22]), and the results are in good agreement. In line (b) of Table II we present an analytical approximation of $h_{0}(\Gamma)$, which follows from the recent MC results of DeWitt and Slattery 22] for a Coulomb liquid. It seems to be the best available evaluation of $h_{0}(\Gamma)$, which is in very good agreement with the expression in line (a). In the indicated $\Gamma$-range, it is accurately approximated by a linear function $h_{0}(\Gamma) \approx 0.9\left(2^{5 / 3}-2\right) \Gamma=1.0573 \Gamma$ suggested by 
TABLE II: Function $h_{0}(\Gamma)$ as calculated by different authors.

\begin{tabular}{llll}
\hline Line & \multicolumn{1}{c}{ Ref. } & \multicolumn{1}{c}{$h_{0}(\Gamma)$} & $\Gamma$ \\
\hline (a) & Eq. (17) in $[16]$ & $1.0531 \Gamma+2.2931 \Gamma^{1 / 4}-0.5551 \ln \Gamma-2.35$ & $1 \leq \Gamma \leq 155$ \\
(b) & Eq. (20) in [10] & $1.0563 \Gamma+1.0208 \Gamma^{0.3231}-0.2748 \ln \Gamma-1.0843$ & $1 \leq \Gamma \leq 170$ \\
(c) & Eq. (6) in [17] & $1.148 \Gamma-0.00944 \Gamma \ln \Gamma-0.000168 \Gamma(\ln \Gamma)^{2}$ & $5 \leq \Gamma \lesssim 180$ \\
(d) & Eq. (19) in [18] & $1.132 \Gamma-0.0094 \Gamma \ln \Gamma$ & $1 \lesssim \Gamma \lesssim 170$ \\
\hline
\end{tabular}

Salpeter [12] using a simple ion-sphere model. At $\Gamma \gg 1$ the plasma screening enhancement is huge. For instance, $\exp \left(h_{0}\right) \sim 10^{74}$ for $\Gamma \sim 170$.

Some authors calculated $H(0)$ and the related enhancement factor $\exp \left(h_{0}\right)$ by extrapolating MC $H(r)$ to $r \rightarrow 0$ (as mentioned above). In particular, Ogata et al. 17, 23] used that formalism to analyze the enhancement of nuclear reactions in one-component and two-component strongly coupled ion liquids. Their $h_{0}(\Gamma)$ is given in line (c) of Table III These calculations are less accurate than those based on Eq. (10) because of the problems of extrapolation of $H(r)$ to $r \rightarrow 0$ in Refs. [17, 23] (see Refs. [10, 11, 15] for details).

The function $h_{0}(\Gamma)$ was also calculated by Ogata 18 using the PIMC method. His result [line (d) in Table I] is in better agreement with the most accurate result [line (b)] as discussed in Ref. [10].

In addition to $\exp \left(h_{0}\right)$, the enhancement factor $F_{\text {scr }}$ in Eq. (9) contains a factor $\exp \left(h_{1}\right)$ which depends on two arguments (e.g., $\Gamma$ and $\zeta$ ). The basic term in $h_{1}$ in the thermonuclear regime with strong screening was obtained by Jancovici [16]; it is presented in line (A) of Table III, where $\zeta$ is given by Eq. (3). Introducing $\tau$ from Eq. (6) we have $\zeta=3 \Gamma / \tau \approx r_{t} / a \sim\left(T_{p} / T\right)^{2 / 3}, r_{t}$ being the Coulomb tunneling length in the thermonuclear regime $\left(T \gtrsim T_{p}\right)$. Clearly, $\zeta$ can be regarded as a small parameter in that regime. This basic term can be easily obtained in the mean-field approximation (Sec. IVB) with the lowest-order expansion terms of $H(r)$ over $r$, $H(r)=H(0)-\frac{1}{4}(r / a)^{2} Z^{2} e^{2} / a$. Treating the $r^{2}$ correction as small and using the semi-classical approximation for the tunneling probability, one immediately comes to Eq. (A).

Equation (A) in Table III can be treated as the well defined lowest-order term in the expansion of $h_{1}(\Gamma, \zeta)$ in powers of $\zeta$. There were several attempts to improve Eq. (A) by adding new terms obtained either on theoretical grounds or by fitting numerical results. These new terms are model dependent and debatable. It is thought that adding these terms allows one to extend the results to lower temperatures, somewhat beyond the lowest boundary $T \sim T_{p}$ for the thermonuclear regime (let us remark that $T=T_{p}$ corresponds to $\zeta=0.513$, and $T=T_{p} / 2$ corresponds to $\zeta=0.815$ ). We will discuss the validity of such extensions in Sec. $\mathrm{V}$

Alastuey and Jancovici 24] proposed semi-analytic corrections to (A). Their result is given in line (B) of
Table III. Ogata et al. [17, 23] calculated the Coulomb tunneling probability and $h_{1}(\Gamma, \zeta)$ using the mean-field potential and solving numerically an effective radial Schrödinger equation. A fit to their calculations is given in line (C). These results were used by Kitamura 25] for constructing an analytic expression for nuclear reaction rates in all burning regimes. Ogata [18] calculated $h_{1}(\Gamma, \zeta)$ using PIMC. His fit is presented in line (D). Itoh et al. [19] determined the enhancement factors $F_{\text {scr }}$ calculating the WKB Coulomb barrier penetrability in a mean-field potential. Their results are equivalent to $h_{1}(\Gamma, \zeta)=1.25 \Gamma-\tau f(\zeta)-h_{0}(\Gamma)$, where $h_{0}(\Gamma)$ is given by Eq. (a) of Table $\amalg$ and $f(\zeta)$ is given by their lengthy fit expression (4.4) [with our $\zeta$ denoted by $\beta$ and $\zeta \leq 5.4$ ]. Let us remark that their mean-field potential $H(r)$ is simplified. Its $H(0)$ value is correct but small- $r$ behavior is approximate (a continuous function with a break).

Recent PIMC calculations by Militzer and Pollock [9] will be analyzed in Sec. V]

\section{Zero-temperature pycnonuclear burning}

Zero-temperature pycnonuclear regime (regime $\mathrm{V}$ in Table (1) takes place at low temperatures, $T \lesssim T_{q}$, particularly, at $T=0 ; T_{q}$ is defined by Eq. (44) and plotted in Fig. 1. In this case, one can safely assume that all plasma ions occupy ground states in their potential wells. Quantum tunneling and nuclear fusion occur mainly between close neighbors owing to zero-point ion vibrations; thermal effects in ion motion are unimportant and the reaction rate is temperature-independent. The reaction rate increases with growing $\rho$ because zero-point vibrations become more efficient.

Coulomb tunneling probability in this regime has been calculated in various approximations (see Refs. 10, 11] for a recent analysis of the results). The predicted reaction rates have similar density dependence but differ within several orders of magnitude.

\section{E. Thermally enhanced pycnonuclear burning}

Thermally enhanced pycnonuclear regime (regime IV from Table (I) occurs at higher temperatures, $T_{q} \lesssim T \lesssim$ $0.5 T_{p}$. These temperatures are still so low that the majority of ions occupy their ground states. However, some 
TABLE III: Function $h_{1}(\Gamma, \zeta)$ as calculated by different authors.

\begin{tabular}{lcllll}
\hline Line & Ref. & \multicolumn{1}{c}{$h_{1}(\Gamma, \zeta)$} & $\Gamma$ & $\zeta$ & $T / T_{p}$ \\
\hline (A) & Eq. (35) in $[16]$ & $-(5 / 32) \Gamma \zeta^{2}$ & $1 \leq \Gamma \leq 155$ & $\zeta \lesssim 1$ & $T / T_{p} \gtrsim 0.37$ \\
(B) & Eq. (28) in [24] & $-(5 / 32) \Gamma \zeta^{2}+0.014 \Gamma \zeta^{3}+0.0128 \Gamma \zeta^{4}$ & $1 \leq \Gamma \leq 155$ & $\zeta \leq 1.6$ & $T / T_{p}>0.18$ \\
(C) & Eq. (36) in $[17]$ & $-(5 / 32) \Gamma \zeta^{2}\left[1+(1.1858-0.2472 \log \Gamma) \zeta-0.07009 \zeta^{2}\right]$ & $5 \leq \Gamma \lesssim 180$ & $\zeta \lesssim 2$ & $T / T_{p} \gtrsim 0.13$ \\
(D) & Eq. (19) in [18] & $-(5 / 32) \Gamma \zeta^{2}\left(1-0.0348 \zeta-0.1388 \zeta^{2}+0.0222 \zeta^{3}\right)$ & $1 \lesssim \Gamma \lesssim 170$ & $\zeta \lesssim 2$ & $T / T_{p} \gtrsim 0.13$ \\
\hline
\end{tabular}

of them populate higher bound energy levels in the potential wells. These ions give the major contribution to the reaction rate because it is much easier for them to penetrate through the Coulomb barrier.

This burning regime was studied in Refs. 8, 26]. As discussed in Ref. [10], the results differ within several orders of magnitude and have to be improved.

When the density grows up, the upper and lower boundary temperatures for this regime, $0.5 T_{p}$ and $T_{q}$, become closer (Fig. 11) and finally merge. Therefore, this reaction regime disappears at sufficiently high densities (when spacings $\sim \hbar \omega_{p}$ between quantum energy levels in potential wells become too large). As a rule, such a density is too high to be of practical importance (it would be $\sim 10^{15} \mathrm{~g} \mathrm{~cm}^{-3}$ for carbon burning, in which case no carbon can survive in dense matter).

\section{F. Thermo-pycnonuclear burning}

This regime (regime III from Table I) takes place at $T_{p} / 2 \lesssim T \lesssim T_{p}$. It is intermediate between thermonuclear regimes and pycnonuclear ones. When the temperature increases from $\sim T_{p} / 2$ to $\sim T_{p}$, those ions, which give the most important contribution into the reaction rate, become unbound and move to continuum states (from closest-neighbor collisions in the pycnonuclear case to collisions of freely moving particles in the thermonuclear case).

\section{G. All regimes and problems}

Several works [10, 11, 25] have suggested analytic fits for the nuclear reaction rates valid in all five burning regimes. In particular, the fits constructed in Refs. 10, 11] take into account current theoretical uncertainties of the reaction rates and give the optimal, maximum and minimum theoretical rates.

In Fig. 1] we present the lines which divide the $T-\rho$ diagram into five regions appropriate to five regimes. In addition, we plot two lines [10] along which the characteristic carbon burning times $\tau_{\text {burn }}=n_{i} / R$ are equal to $1 \mathrm{~s}$ and $10^{10}$ years (the upper and lower lines, respectively). The astrophysical factor $S(E)$ for the carbon burning is taken from Ref. [10]. (We neglect a possible hindrance of the carbon reaction [27] at energies much lower than the Coulomb barrier energy.) The lines are almost horizontal at lower $\rho$ and higher $T$, where carbon burns in thermonuclear regimes, and they are almost vertical at higher $\rho$ and lower $T$, where carbon burns in pycnonuclear regimes. Above and to the right of the $\tau_{\text {burn }}=1 \mathrm{~s}$ line the burning is very fast, and there is no carbon in dense matter. Below and to the left of the $\tau_{\text {burn }}=10^{10} \mathrm{yr}$ line the burning is extremely slow (practically absent). Therefore, the density-temperature domain of practical interest for carbon burning is located between these two lines.

Although the main features of the Coulomb tunneling in dense matter seem clear, some important tunneling problems in OCP are still unsolved:

(i) In the thermonuclear regime, where the plasma screening is conveniently described by the enhancement factor $F_{\text {scr }}$ [Eqs. (7) and (9)], the function $h_{0}(\Gamma)$ is well defined [line (b) of Table II. But what is an exact form of the function $h_{1}(\Gamma, \zeta)$ (Table III)?

(ii) Down to which temperatures this description can be extended?

(iii) What is an exact expression for the reaction rate at lower temperatures when the burning becomes pycnonuclear?

Our aim will be to analyse problems (i) and (ii) and discuss (iii). The problems in multicomponent ion mixtures are more complicated [11].

\section{MILITZER-POLLOCK PIMC CALCULATIONS}

New PIMC calculations of Militzer and Pollock [9] were performed for a wide range of plasma parameters which cover all regimes of nuclear burning. In Fig. 1 filled dots show some densities and temperatures of stellar matter which correspond to four Militzer-Pollock points (the values of $\Gamma$ and $r_{s}$ in their Table 1) if applied for carbon burning. The majority of their points are not shown; they would refer to much higher $T$ and $\rho$ than those displayed in Fig. 1 (far from the $T-\rho$ region where carbon can exist in dense stellar matter). 
In principle, the PIMC is the best method to calculate Coulomb tunneling in nuclear reactions. It can take into account all the effects of dense environment on Coulomb tunneling, including fluctuative nature of plasma potential; dynamical response of plasma ions to the motion of reacting nuclei in the course of quantum tunneling; finite width of trajectories of the tunneling nuclei (that is beyond the WKB approach). Unfortunately, highly accurate PIMC calculations require huge computer resources (long PIMC runs with many plasma ions involved) while all PIMC calculations performed so far are naturally limited, at least by not too many plasma ions.

Notice that the PIMC simulations [9] neglected the effects of quantum statistics of ions. As shown by several authors (e.g., Ref. [18]), this is a good approximation for the conditions of practical interest. Usually, nuclear burning occurs when $\rho$ is still insufficiently high for the quantum statistics effects to be pronounced.

PIMC simulations of Militzer and Pollock [9] included 54 plasma ions. The authors calculated the contact probabilities $g(0)$, which are the values of the quantummechanical radial pair distribution function $g(r)$ at $r \rightarrow$ 0 . For OCP of ions, $g(0)$ is related to the reaction rate through [28]

$$
R=\frac{n_{i}^{2}}{\pi} \frac{a_{B}}{\hbar} S\left(E_{\mathrm{pk}}\right) g(0),
$$

where $a_{B}=\hbar^{2} /\left(m_{i} Z^{2} e^{2}\right)$.

Militzer and Pollock present their $g(0) \equiv g_{M P}(0)$ for 36 values of plasma parameters $\Gamma$ and $\eta$ (equivalently, for 36 values of $\rho$ and $T$, four of which are shown in Fig. 11). Specifically, they considered 10 values of $\Gamma$ : $\Gamma=0.5$ (five $\eta$-points), $\Gamma=1$ (five points), $\Gamma=2$ (five points), $\Gamma=5$ (five points), $\Gamma=10$ (five points), $\Gamma=40$ (five points), $\Gamma=100$ (three points), $\Gamma=200$ (one point), $\Gamma=400$ (one point), and $\Gamma=600$ (one point). All their results are shown in Fig. 2, where we compare them with our calculations in the mean-field WKB approximation $\left[g(0)=g_{\mathrm{MF}}(0)\right.$; see Secs. IV and V] below]. Note five typos in Table I of Ref. [9]; in the values of $-\ln [g(0)]$ in column 5 for $\eta=0.25, \Gamma=100 ; \eta=0.5, \Gamma=100$; and $\eta=1, \Gamma=200,400$ and 600 , one should remove zero after dot; this removal restores correct errobars of $-\ln [g(0)]$ (otherwise the errorbars are ten times smaller than their actual values).

We have divided the Militzer-Pollock data (somewhat arbitrarily) into three groups (i), (ii) and (iii) (listed in Table IV]).

The first group (i) consists of five points with $\Gamma=0.5$ (marked by squares in Fig. 2). These points correspond to a moderately strong Coulomb coupling $\left(T=2 T_{l}\right.$, thermonuclear burning intermediate between weak and strong plasma screening regimes). We do not analyze them in detail because in this case the effects of plasma screening on Coulomb tunneling are weak and exact screening enhancement factors have not been calculated so far by other theoretical methods.
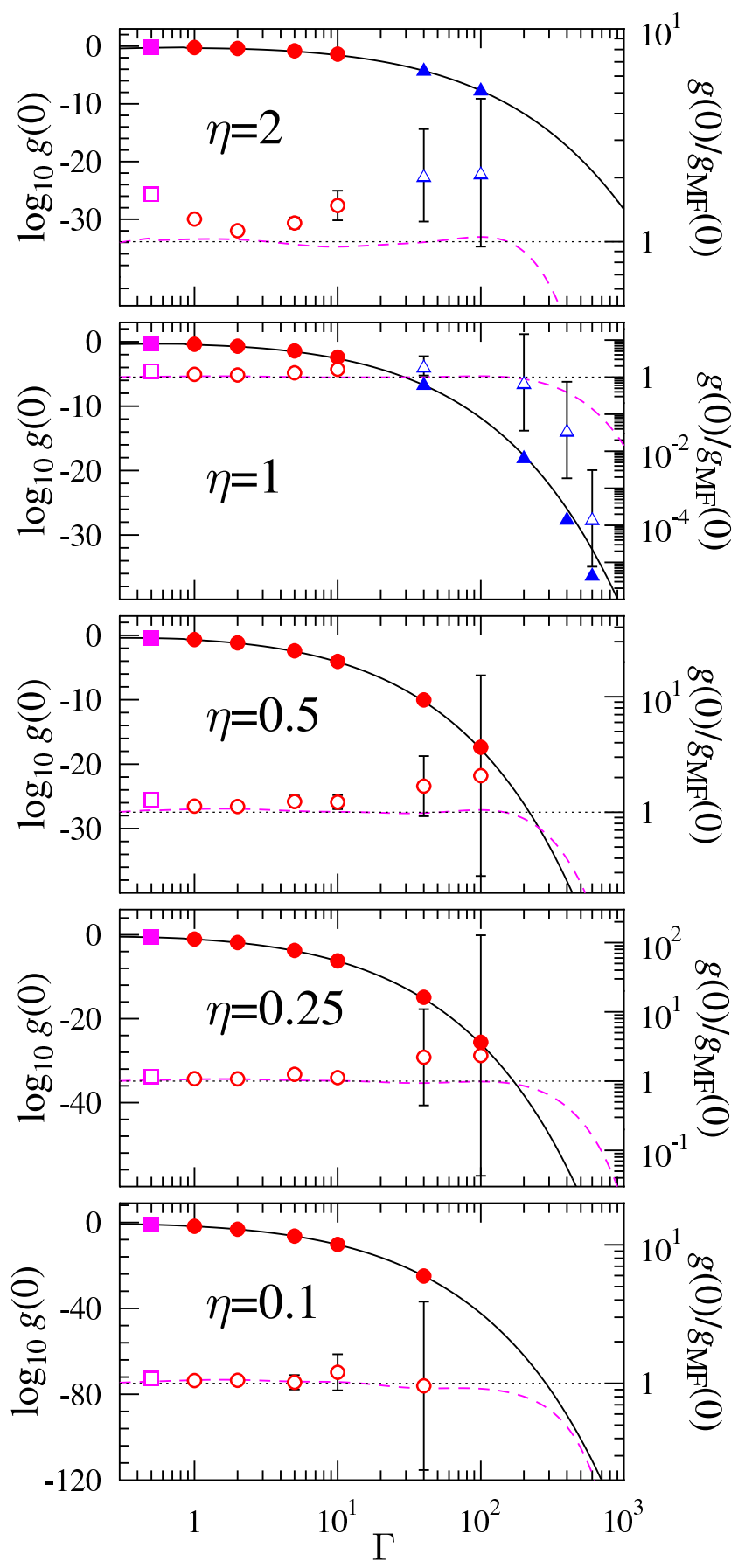

FIG. 2: Contact probabilities versus $\Gamma$ for five values of $\eta=0.1$, $0.25,0.5,1$, and 2 . Left vertical axis: Filled symbols show all Militzer-Pollock $g_{M P}(0)$ data; squares, dots and triangles mark the data of the three groups (i)-(iii) (Table IV). Solid lines are the contact probabilities $g_{\mathrm{MF}}(0)$ calculated in the mean-field WKB approximation. Right vertical axis: Open symbols with errorbars display the ratio $g_{M P}(0) / g_{\mathrm{MF}}(0)$ of the PIMC to mean-field WKB results [dotted lines refer to $g_{M P}(0)=g_{\mathrm{MF}}(0)$ to guide the eye]. Dashed lines show the ratio $g_{\mathrm{MF}}^{\text {fit }}(0) / g_{\mathrm{MF}}(0)$ of our mean-field WKB fitted (Sec. IV C) and calculated values. See text for details. 
TABLE IV: Three groups of Militzer-Pollock data points [9].

\begin{tabular}{ccc}
\hline Line & Group & Domain \\
\hline (i) & Moderate plasma screening & $\Gamma=0.5$ \\
(ii) & $T$ dependent rate, strong screening & $\Gamma \geq 1, \zeta<3$ \\
(iii) & $T$ independent pycnonuclear data & (see text) \\
\hline
\end{tabular}

The second group (ii) includes 26 data points (dots in Fig. 2) with $1 \leq \Gamma \leq 100$ and $\zeta \leq 3\left(T \geq 0.071 T_{p}\right)$. Three such points belong to the thermonuclear regime with strong screening $\left(T \geq T_{p}\right)$, seven points correspond to the intermediate thermo-pycnonuclear regime $\left(0.5 T_{p}<T<T_{p}\right)$, while other 16 points refer to pycnonuclear burning at not too low $T\left(0.071 T_{p} \leq T \leq 0.5 T_{p}\right)$.

The third group (iii) includes six points (triangles in Fig. 2). Three of them have very large $\Gamma=200,400$ and 600 (with $\zeta=4.33,5.45$, and 6.24 , respectively). The other three have lower $\Gamma=40,40$, and 100 but large $\zeta(2.53,3.18$, and 4.32$)$, i.e., small $T \leq 0.0912 T_{p}$ at which the contact probability is expected to become temperature independent. The point with $\Gamma=40$ and $\zeta=2.53$ belongs also to the second group.

\section{MEAN FIELD WKB APPROXIMATION}

In this section we calculate the nuclear reaction rate in a mean-field WKB approximation, which is much simpler than the PIMC.

\section{A. Mean-field potential}

We start with the discussion of the mean field potential $H(r)$ in a strongly coupled OCP. We have taken the results of extensive Monte Carlo (MC) calculations of $H(r)$ for a classical OCP of ions in a liquid phase for ten values of $\Gamma$ from 1 to 200 within some intervals of $x=r / a$ (Fig. 3). Although a thermodynamically stable phase of OCP at $\Gamma>175$ is a crystalline bcc lattice, the difference of free energies of the lattice and liquid is small. As a result, the OCP can stay liquid at temperatures much below the melting point, and one can simulate supercooled liquid in MC runs. In order to check our results we have also taken MC calculations of $H(r)$ for a classical bcc Coulomb crystal at $\Gamma=400$ and 800 (two last panels in Fig. 3).

It is useful to introduce a dimensionless mean-field potential $u(r)$,

$$
H(r) / k_{B} T=\Gamma u(x),
$$

which is plotted in Fig. 3 .

The dimensionless potential $u(x)$ depends on two variables, $x$ and $\Gamma$. For further use, we have fitted the calcu-

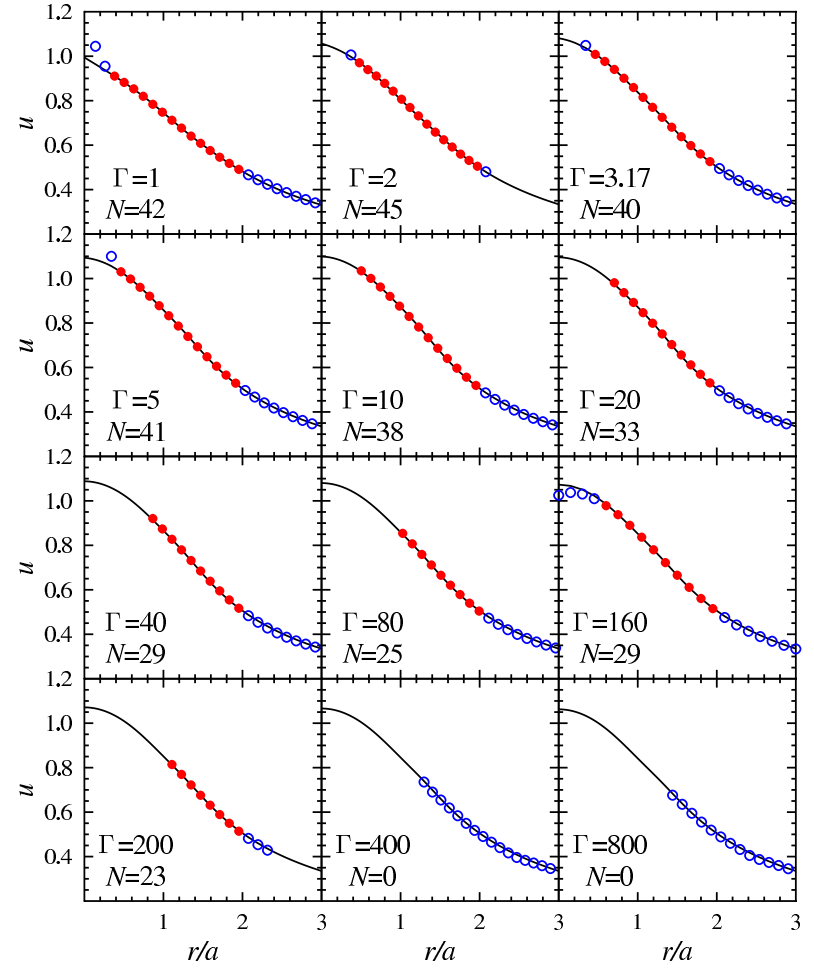

FIG. 3: (Color online) Normalized mean field MC potential $u(x)$ in an OCP of ions for 12 values of $\Gamma$ from 1 to 800 . The data for $\Gamma \leq 200$ are obtained for ion liquid, while the data for $\Gamma=400$ and 800 correspond to bcc ion crystal. Filled and open dots show data points included into fitting and excluded from it, respectively. Lines show the fit; $N$ is the number of data points included into the fitting. See text for details.

lated values of $u(x)$ by an analytical expression. The fitting included selected MC points (denoted by filled dots) for an OCP liquid at $1 \leq \Gamma \leq 200$. The number $N$ of these data points at any $\Gamma$ is presented in Fig. 3 (for a better visualization we have not plotted some points). Other MC points, not included into the fitting, are denoted by open dots. In particular, we did not fit the data for the ion crystal $(\Gamma=400$ and 800$)$. We have also excluded from the fitting several data points at low $x$ for $\Gamma \leq 5$ and $\Gamma=160$. These points were calculated with large errors because of poor MC statistics at low separations $x$. We do not show their errorbars to simplify the figure. The errorbars for all other points are small and would be invisible. The data points at sufficiently large $x$ for $\Gamma \leq 200$, excluded from the fitting, have been used for checking the quality of our fit. Similar potentials for OCP liquid have been computed, e.g., in Ref. [29]. The potentials suggested in Ref. [30] for $4 \leq \Gamma \leq 90$ and $x \lesssim 1.6$ are also very accurate.

To ensure a high fit accuracy at small $x$, where MC statistics is insufficiently good, we have taken into account that at small $x$ the function $u(x)$ can be expanded in powers of $x$. In the limit of strong Coulomb coupling 
$(\Gamma \gg 1)$ only even powers of $x$ should survive [31] and the expansion should have the form

$$
u(x)=\alpha_{0}+\alpha_{2} x^{2}+\alpha_{4} x^{4}+\alpha_{6} x^{6}+\ldots,
$$

where $\alpha_{0}=h_{0}(\Gamma) / \Gamma, \alpha_{2}=-\frac{1}{4}[16]$, and $\alpha_{4}, \alpha_{6}, \ldots$ can depend on $\Gamma$. Thus, $\alpha_{0}$ is a slowly varying function of $\Gamma$ determined by the function $h_{0}(\Gamma)$ (discussed in Sec. IIC). One can use any accurate representation of $h_{0}(\Gamma)$, for instance, Eqs. (a) or (b) from Table II. However, the fit formula (14) for $u(x)$ presented below is especially accurate if $h_{0}(\Gamma)$ is given by our own fit expression (19) (see Sec. IV C). For a not too strong Coulomb coupling ( $\Gamma \lesssim 5$ ), odd powers of $x$ can become pronounced in the expansion (13), and $\alpha_{2}$ can be modified.

We have fitted our MC data points by the analytic expression

$$
u(x)=\alpha_{0}\left[\frac{1-C_{4} x-2\left(C_{1} / \alpha_{0}\right) x^{2}+C_{3} x^{4}+C_{2} x^{8}}{1+C_{2} \alpha_{0}^{2} x^{10}}\right]^{1 / 2},
$$

where

$$
\begin{array}{ll}
C_{1}=0.25-0.267 \Gamma^{-1.44}, & C_{2}=0.05 \\
C_{3}=0.084-0.144 \Gamma^{-1.7}, & C_{4}=0.434 \Gamma^{-1.2} .
\end{array}
$$

The fit quality is demonstrated in Fig. 3. The rootmean-square relative error is $0.4 \%$, the maximum error $1.1 \%$ takes place at $\Gamma=10$ and $x=1.67$. It is seen that the fit is accurate for a classical Coulomb liquid (any $\Gamma \geq 1$ ) at least at $x \lesssim 3$. This is sufficient to calculate the plasma screening enhancement of reaction rates (Sec. IVB). It is remarkable that the fit is valid also in the crystalline solid ( $\Gamma=400$ and 800$)$ although we have not included the crystalline data into the fitting. Clearly, $H(r)$ in the solid and strongly coupled liquid $(\Gamma \gg 1)$ is nearly the same, being "frozen" (almost independent of $\Gamma)$. This allows us to expect that Coulomb tunneling and the fusion reaction rate should not undergo significant changes when the temperature drops below the freezing temperature $T_{m}$.

\section{B. Enhancement factor}

Having $H(r)$ we can introduce the mean-field reaction rate

$$
\begin{aligned}
R^{\mathrm{MF}}= & \frac{n_{i}^{2} S_{\mathrm{pk}}}{2} \sqrt{\frac{8}{\pi \mu\left(k_{B} T\right)^{3}}} \\
& \times \int_{E \min }^{\infty} \mathrm{d} E \exp \left[-\frac{E}{k_{B} T}-P(E)\right],
\end{aligned}
$$

where $E$ is an energy of relative motion of colliding nuclei (with a minimum value $E_{\text {min }}$ at the bottom of the potential well $), \exp \left(-E / k_{B} T\right)$ comes from the Maxwellian energy distribution of the nuclei, and $S_{\mathrm{pk}}$ is the $S$-factor corresponding to the energy $E$ at which the integrand has maximum. Finally,

$$
P(E)=\frac{2 \sqrt{2 \mu}}{\hbar} \int_{r_{n}}^{r_{t}} \mathrm{~d} r \sqrt{\frac{Z^{2} e^{2}}{r}-H(r)-E}
$$

characterizes the penetrability of the Coulomb barrier at an energy $E ; r_{n}$ and $r_{t}$ are classical turning points which are zeros of the expression under the square root; we can set $r_{n} \rightarrow 0$ (an exact $r_{n}$ should have been determined by nuclear interactions which we neglect in the Coulomb tunneling problem). Here we use a radial WKB approximation with the mean-field potential $H(r)$. A similar approach was used by Ogata et al. [17, 23]. The main difference is that Ogata et al. numerically solved a radial Schrödinger equation which should be equivalent to the WKB integration under the conditions of study. Notice that Ogata et al. employed less accurate mean-field potentials and obtained, therefore, less accurate results as discussed e.g., in [10, 15] (also see Sec. IIC). The approach equivalent to our but with a less accurate meanfield potential was used by Itoh et al. [19] (Sec. [ICl).

Putting $H(r)=0$ in Eq. (17) we reproduce the well known result $P(E)=2 \pi Z^{2} e^{2} /(\hbar v)$ for a pure Coulomb barrier (with $v=\sqrt{2 E / \mu}$ ). Taking energy integral in Eq. (16) by a saddle-point method we come to the thermonuclear reaction rate without plasma screening, $R^{\mathrm{MF}}\{H=0\}=R_{\mathrm{th}}$, given by Eq. (5).

In the WKB mean-field approximation the enhancement factor of the nuclear reaction rate is

$$
F_{\mathrm{scr}}^{\mathrm{MF}}=R^{\mathrm{MF}}\{H\} / R^{\mathrm{MF}}\{0\} .
$$

The calculation of $F_{\mathrm{scr}}^{\mathrm{MF}}$ from Eq. (18) reduces to the evaluation of $P(E)$ and $R^{\mathrm{MF}}\{H\}$ from Eqs. (17) and (16) for a given $H(r)$. We have performed the integrations over $r$ and $E$ numerically (beyond the saddle-point approximation) in all integrals.

These results are naturally restricted by the WKB and mean-field approximations. They neglect fluctuative nature of plasma microfields; deviations from spherical symmetry and dynamical evolution of these microfields during a tunneling event; corrections to $H(r)$ due to quantum effects in ion motion; deviations from the first-order one-dimensional (radial) WKB approximation. In the thermonuclear regime with strong plasma screening all these effects are not expected to be strong.

\section{Analytic fit}

To facilitate applications of our results we have fitted the values of the enhancement factor $F_{\mathrm{scr}}^{\mathrm{MF}}$, calculated from Eq. (18), by an analytic expression. First of all, we notice that at $1 \leq \Gamma \leq 200$ the function $h_{0}(\Gamma)$ can be 
approximated as

$$
\begin{aligned}
h_{0}^{\mathrm{fit}}(\Gamma)= & \Gamma^{3 / 2}\left(\frac{A_{1}}{\sqrt{A_{2}+\Gamma}}+\frac{A_{3}}{1+\Gamma}\right) \\
& +\frac{B_{1} \Gamma^{2}}{B_{2}+\Gamma}+\frac{B_{3} \Gamma^{2}}{B_{4}+\Gamma^{2}},
\end{aligned}
$$

with $A_{1}=2.7822, A_{2}=98.34, A_{3}=\sqrt{3}-A_{1} / \sqrt{A_{2}}=$ $1.4515, B_{1}=-1.7476, B_{2}=66.07, B_{3}=1.12$, and $B_{4}=65$. The accuracy of this fit is the same as the accuracy of the best fit (b) in Table III. At $\Gamma \gg 1$ it gives $h_{0}^{\text {fit }}(\Gamma) \approx 1.0346 \Gamma$ and remains accurate at $200 \leq \Gamma \leq 600$. In addition, it reproduces the correct Debye-Hückel asymptote $h_{0}^{\mathrm{fit}}(\Gamma)=\sqrt{3} \Gamma^{3 / 2}$ at $\Gamma \ll 1$. Note that the functional form of Eq. (19) was suggested in Ref. [20] to approximate the free energy of OCP.

We have fitted the values of the enhancement factor, calculated on a dense grid of values $1 \leq \Gamma \leq 200$ and $0 \leq \zeta \leq 8$, as $F_{\mathrm{scr}}^{\mathrm{MF}}=\exp \left(h_{\mathrm{MF}}^{\mathrm{fit}}\right)$,

$$
h_{\mathrm{MF}}^{\mathrm{fit}}(\Gamma, \zeta)=h_{0}^{\mathrm{fit}}(\Gamma)+h_{1}^{\mathrm{fit}}(\Gamma, \zeta)=h_{0}^{\mathrm{fit}}(\widetilde{\Gamma})
$$

with

$$
\widetilde{\Gamma}=\Gamma /\left(1+\alpha \zeta+\beta \zeta^{2}+\gamma \zeta^{3}\right)^{1 / 3},
$$

$\alpha=0.022, \beta=0.41-0.6 / \Gamma$, and $\gamma=0.06+2.2 / \Gamma$. The maximum fit error of $F_{\mathrm{scr}}^{\mathrm{MF}}$ is $\approx 30 \%$; it occurs at $\zeta=0.4$ and $\Gamma=200$, at which the enhancement factor itself is enormously large, $F_{\mathrm{scr}}^{\mathrm{MF}} \sim 10^{90}$. The fit accuracy is illustrated in Fig. 2 where the dashed lines (right vertical scales) show the ratio of fitted and calculated WKB mean-field values of $g_{\mathrm{MF}}(0)$ [same as the ratios of fitted and calculated values of $\left.F_{\mathrm{scr}}^{\mathrm{MF}}\right]$. We have checked that an extension of $\zeta$ from 8 to 50 at $1 \leq \Gamma \leq 200$ does not change the initial fit accuracy. Moreover, if $\zeta \leq 50$, the fit remains sufficiently accurate up to $\Gamma \sim 600$. For instance, at $\Gamma=400$ the fit gives the values of $F_{\mathrm{scr}}^{\mathrm{MF}}$ which differ from the calculated values within a factor of 2 , and at $\Gamma=600$ - within a factor of 10 . If $\Gamma \gg 1$ and $\zeta \lesssim 1$, the main difference between $\widetilde{\Gamma}$ and $\Gamma$ in Eq. (21) is determined by the term in the denominator containing $\beta \approx 0.41$ (while the terms containing $\alpha$ and $\gamma$ are relatively small). Neglecting $\alpha$ and $\gamma$ for a moment and treating $\beta \zeta^{2}$ as a small correction, we obtain $h_{1} \approx-1.0346 \times 0.41 \Gamma \zeta^{2} / 3 \approx-0.141 \Gamma \zeta^{2}$, which is very close to $-(5 / 32) \Gamma \zeta^{2} \approx-0.156 \Gamma \zeta^{2}$ given by Eq. (A) of Table III and discussed in Sec. IIC

To summarize, our fit gives very accurate values of $F_{\mathrm{scr}}^{\mathrm{MF}}$ for all possible values of $\Gamma$ and $\zeta$ at which the WKB mean-field approximation is valid (see below) and, actually, in much wider domain.

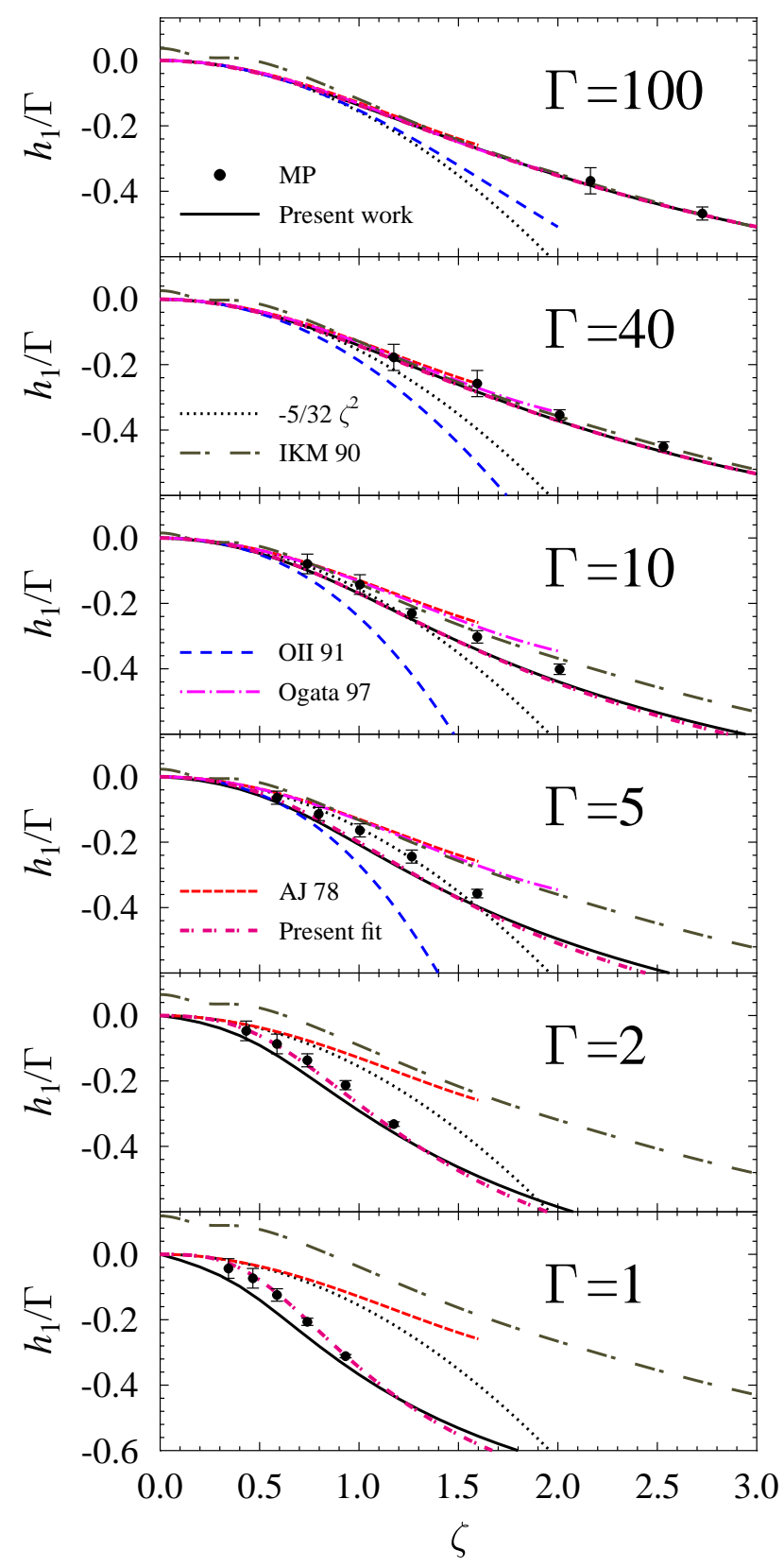

FIG. 4: (Color online) The screening function $h_{1}^{\mathrm{MF}}(\Gamma, \zeta) / \Gamma$ versus $\zeta$ at six values of $\Gamma$. Dots with errorbars are MilitzerPollock points [of group (ii) in Table IV], solid lines are our mean-field WKB calculations, and short dash-dot-space lines are given by our fit. Dotted lines show the lowest-order result of Jancovici [16], while short-dash, long dash-dot-space, longdash, and dot-dash lines present the results of Alastuey and Jancovici (AJ 78, Ref. 24]), Itoh et al. (IKM 90, Ref. [19]), Ogata et al. (OII 91, Ref. [17]), and Ogata (Ogata 97, Ref. [18]), respectively. 


\section{PIMC, MEAN-FIELD WKB, AND OTHER RESULTS}

\section{A. Overall analysis}

After calculating $F_{\text {scr }}^{\mathrm{MF}}$ we have used Eq. (11) and determined $g_{\mathrm{MF}}(0)$. The results are shown by solid lines in Fig. 2 for the same five values of $\eta$ which were taken by Militzer and Pollock [9]. This allows us to directly compare the PIMC and mean-field WKB approaches for all PIMC points [all groups (i)-(iii) of data points in Table IV. Open symbols (right vertical scale) show ratios of the Militzer-Pollock to calculated mean-field results. The errorbars are those as reported [9] in the PIMC simulations of $g_{M P}(0)$ (with the corrections mentioned in Sec. III). The overall agreement seems very satisfactory. Large differences take place in pycnonuclear points. Very strong differences $g_{M P}(0) / g_{\mathrm{MF}}(0) \sim 0.04$ and $\sim 10^{-4}$ occur for $\eta=1$ at $\Gamma=400$ and 600 , respectively. We analyze all these results below.

\section{B. Data of group (ii)}

After calculating the enhancement factor $F_{\text {scr }}^{\mathrm{MF}}$, we have presented it in the form (7) and determined $h^{\mathrm{MF}}$. Using then Eqs. (9) and (19) we have calculated $h_{1}^{\mathrm{MF}}=$ $h^{\mathrm{MF}}-h_{0}(\Gamma)$. This function is not very certain and has been a subject of debates (Secs. IIC and IIG). Solid lines in Fig. 4 show our calculated values of $h_{1}(\Gamma, \zeta) / \Gamma$ versus $\zeta$ for six values of $\Gamma=1,2,5,10,40$, and 100 . Short dash-dot-space lines are given by our fit expression (Sec. IVC).

Furthermore, taking the contact probabilities calculated by Militzer and Pollock in the points which belong to group (ii) (Table IV) and using Eq. (11) we have determined the PIMC values of $h_{1}(\Gamma, \zeta)$ for the same six values of $\Gamma$ as in Fig. 4 at several values of $\zeta$. These data are plotted in Fig. 4 by dots, together with numerical errorbars of Militzer and Pollock [9]. One can observe a remarkably good agreement between the meanfield WKB and PIMC results for the data of group (ii). Strongest disagreement occurs at the lowest $\Gamma=1$, where the function $h_{1}$ introduces small contribution into the plasma screening enhancement of the nuclear reaction rates. The existence of real disagreement at $\Gamma \sim 1$ between the PIMC and the mean-field WKB results could be checked in future more extensive PIMC runs. If real, this disagreement could be attributed to a fluctuative nature of the plasma potential at $\Gamma \lesssim 1$ (where Coulomb coupling is not too strong and can allow noticeable fluctuations of the plasma potential from its mean-field values). This would indicate that the PIMC results are more accurate at $\Gamma \lesssim 1$ than the WKB mean-field results.

We have also compared the Militzer-Pollock and our results with some other calculations of $h_{1}(\Gamma, \zeta)$ discussed in Sec. IIC In particular, the dotted lines in Fig. 4 show the basic lowest-order expression (A) from Table III ob-

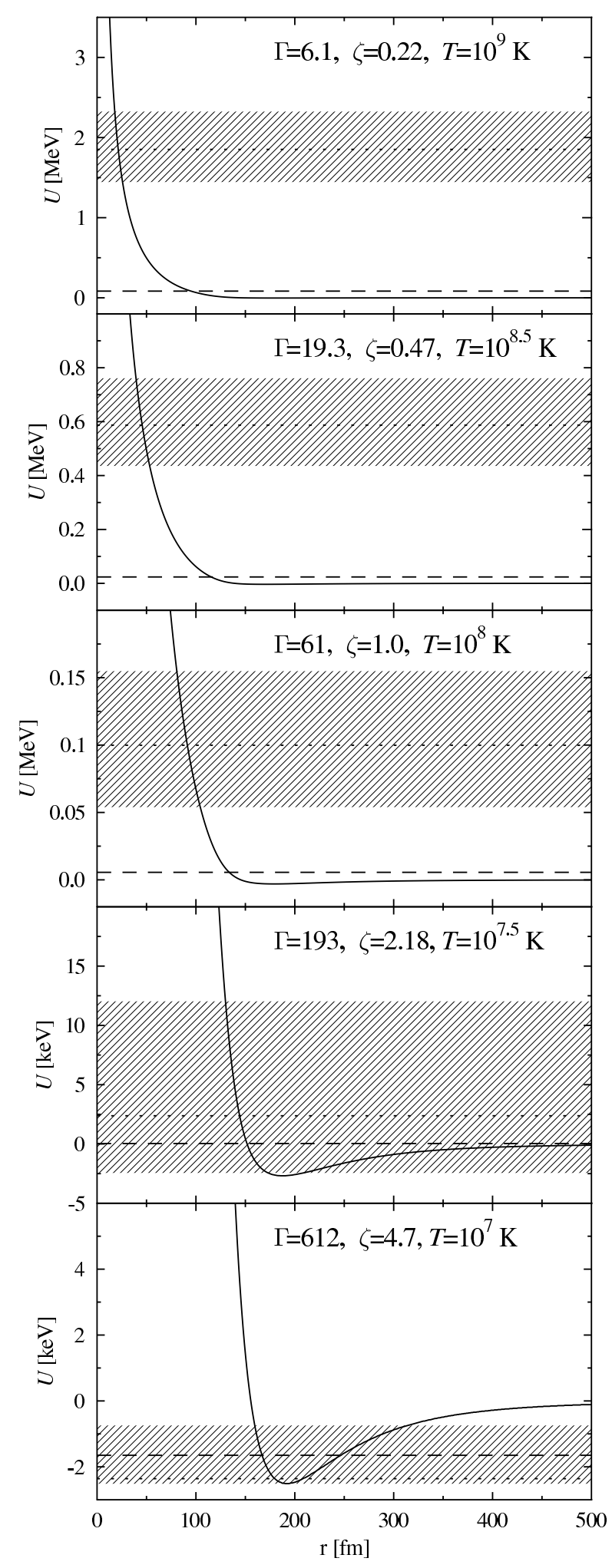

FIG. 5: Effective mean-field Coulomb potential $U(r)$ for reacting carbon nuclei at $\rho=5 \times 10^{9} \mathrm{~g} \mathrm{~cm}^{-3}$ and five temperatures $\left(\log _{10} T[\mathrm{~K}]=9,8.5,8,7.5\right.$, and 7$)$. Shaded strips show Gamow-peak energy ranges; dotted lines are Gamow-peak energies; dashed lines are thermal energies $k_{B} T$ measured from the bottom of $U(r)$. 


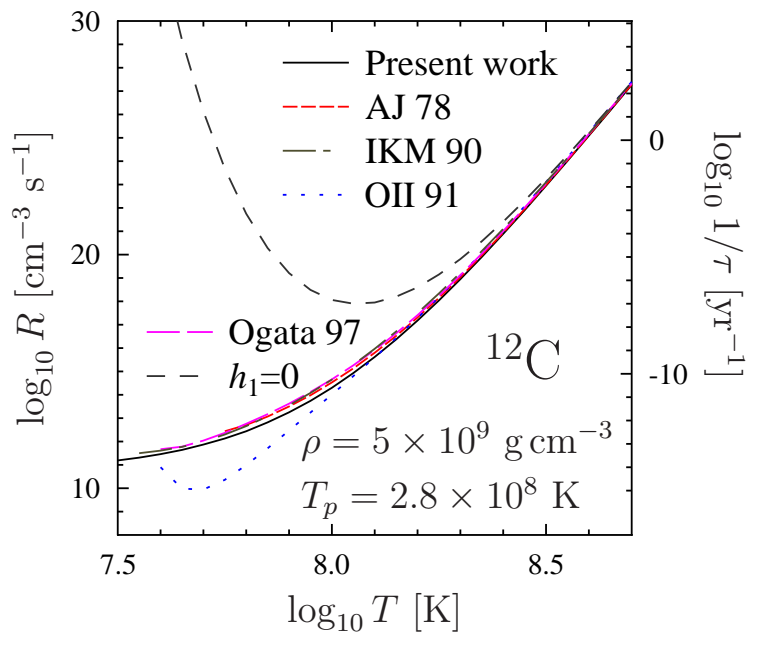

FIG. 6: (Color online) Temperature dependence of the carbon burning rate $R$ (left vertical scale) and the inverse carbon burning time $\tau^{-1}=R / n_{i}$ (right vertical scale) at $\rho=5 \times 10^{9}$ $\mathrm{g} \mathrm{cm}^{-3}$ for the temperature range $T \gtrsim T_{p}$ of thermonuclear burning with strong screening and for lower $T$ (down to $\left.T \sim 0.1 T_{p}\right)$. The upper line is obtained with the screening enhancement function $h=h_{0}(\Gamma)$ given by Eq. (b) in Table II Other lines employ the total function $h=h_{0}+h_{1}$ calculated by Alastuey and Jancovici (AJ 78, Ref. 24]), Itoh et al. (IKM 90, Ref. [19]), Ogata et al. (OII 91, Ref. [17]), and Ogata (Ogata 97, Ref. [18]). The solid line plots our WKB mean-field calculation. See text for details.

tained by Jancovici [16]; the short-dashed, long-dashed, and dot-dashed lines display the expressions (B), (C), and (D) calculated, respectively, by Alastuey and Jancovici [24], Ogata et al. [17], and Ogata [18]. These results are shown for those ranges of $\Gamma$ and $\zeta$ for which they were obtained in the cited publications (Table III). In addition, long-dash-dot-space lines present the function $h_{1}(\Gamma, \zeta)$ which corresponds to the results of Itoh et al. 19] (discussed in Sec. IIC). We see that the MilitzerPollock and our results are in good agreement with earlier predictions of Jancovici, and especially of Alastuey and Jancovici, and Ogata [18], but they are in worse agreement with the results of Ref. 17]. Calculations of Ref. [19] are also accurate; some whirls of corresponding curves at small $\zeta$ occur possibly because of simplified approximation of the mean potential in the cited publication (see Sec. IIC).

As a byproduct of our calculations in the mean-field WKB approximation, we have determined the energy $E_{\mathrm{pk}}$ of the peak of the integrand function in Eq. (16); it is the Gamow-peak energy modified by the plasma screening effects. We have also estimated characteristic energy widths of the Gamow peak (at the half width of the peak maximum). In Fig. 5 we show the effective total radial mean-field Coulomb potential $U(r)=Z^{2} e^{2} / r-H(r)$ for ${ }^{12} \mathrm{C}$ ions reacting in pure carbon matter at $\rho=5 \times 10^{9}$ $\mathrm{g} \mathrm{cm}^{-3}$. At this $\rho$, the ion-sphere radius is $a=98 \mathrm{fm}$. Naturally, $U(r)$ has a minimum at $r \approx 2 a$ due to Coulomb coupling. We plot $U(r)$ for five temperatures, $T=10^{9}$, $10^{8.5}, 10^{8}, 10^{7.5}$, and $10^{7} \mathrm{~K}$. With decreasing $T$, the minimum becomes more pronounced and finally "freezes" at $\Gamma \gtrsim 100$. The shaded strips in Fig. 5 show the Gamowpeak energy ranges and the dotted lines show $E_{\mathrm{pk}}$. The dashed lines present the thermal energy level $k_{B} T$ measured from the bottom of $U(r)$.

The first two upper panels in Fig. 15 refer to the thermonuclear reaction regime with strong plasma screening $\left(T \gtrsim T_{p}\right)$. The next two panels are for a colder plasma ( $T=0.368 T_{p}$ and $0.114 T_{p}$, respectively), while the lowest panel is for a very cold plasma $\left(T=0.0361 T_{p}\right)$, certainly in the zero-temperature pycnonuclear regime. When the temperature decreases, the Gamow-peak energy range becomes thinner (note the difference of energy scales in different panels!) and shrinks to lower energies, together with $E_{\mathrm{pk}}$. In the three upper panels the Gamow peak range is still at $E>0$ [belonging to continuum states in a potential $U(r)$ ]. The energies from this range are much higher than $k_{B} T$ supporting the statement that the main contribution into reaction rates at sufficiently high $T$ comes from suprathermal ions. In these cases, the underlying mean-field WKB approximation can be adequate. In the forth panel, the lowest energies of the Gamow-peak range become negative (drop to bound states) although $E_{\mathrm{pk}}$ is still positive and higher than $k_{B} T$. The mean-field WKB approach may be qualitatively correct but quantitatively inaccurate. At the bottom panel the Gamowpeak energy range fully shrinks to bound-state energies and the formal Gamow-peak energy becomes lower than $k_{B} T$. It is clear that the mean-field WKB approximation breaks down at these low temperatures, and the formally calculated $E_{\mathrm{pk}}$ is inaccurate. Therefore, it is natural that the mean-field WKB results diverge from the PIMC ones at low temperatures. This divergence is seen in Fig. 2 (at highest values of $\Gamma$, especially at $\Gamma=400$ and 600 for $\eta=1$ ).

As follows from the above consideration, the meanfield WKB approximation can be valid for $\zeta \lesssim 1.6-3$ $\left[T \gtrsim(0.1-0.2) T_{p}\right]$. This is further illustrated in Fig. [6 which shows the carbon burning rate versus temperature at the same density $\rho=5 \times 10^{9} \mathrm{~g} \mathrm{~cm}^{-3}$ as in Fig. 5 The upper line is obtained with the simplified enhancement factor $F_{\text {scr }}=\exp \left[h_{0}(\Gamma)\right]$, where $h_{0}(\Gamma)$ is given by Eq. (b) in Table II. It is seen to be a good approximation in the thermonuclear regime $\left(T \gtrsim T_{p}\right)$ but gives qualitatively wrong results just after $T$ decreases below $T_{p}$. It is easy to check that in the thermonuclear regime with strong screening $h_{1}$ is indeed a small correction to $h_{0}$.

However, adding $h_{1}$ and using $F_{\text {scr }}=\exp \left(h_{0}+h_{1}\right)$ greatly helps extending the strong-screening thermonuclear results to lower temperatures, down to $T \sim(0.1-$ 0.2) $T_{p}$. Below $T_{p}$ the function $h_{1}$ is no longer small but becomes comparable to $h_{0}$ and crucial to get physically reasonable results. All other lines in Fig. 6 are plotted by adding $h_{1}$ calculated in the various approximations. The solid line shows our mean-field WKB calculations which are nearly identical to our fit and to the Militzer-Pollock 


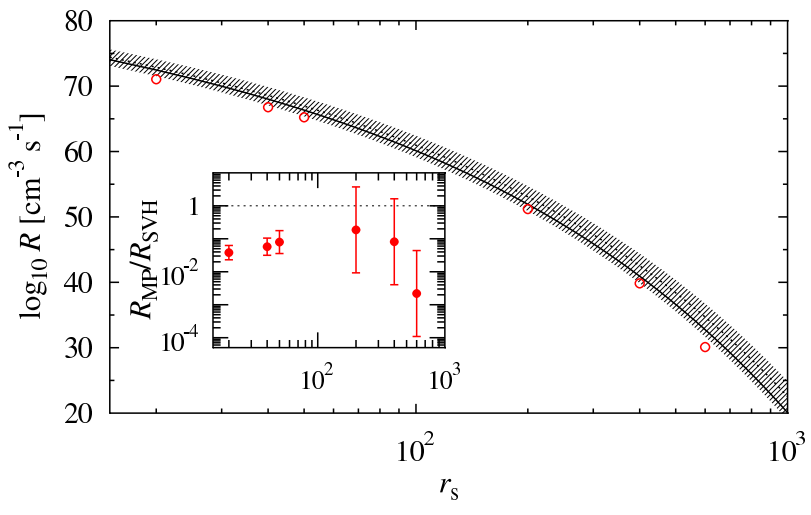

FIG. 7: Pycnonuclear temperature-independent carbon burning rate versus the density parameter $r_{s}$. Open dots show Militzer-Pollock points. Shaded strip presents theoretical uncertainties of other calculations and solid line is the optimal model among these calculations [10]. Filled circles with errorbars in the inset give the ratio of the Militzer-Pollock rates to the predictions of the optimal (Salpeter and Van Horn, SVH) model.

PIMC results in the displayed temperature range. The short-dashed line corresponds to $h_{1}$ and $h_{0}$ obtained by Jancovici [16] and Alastuey and Jancovici 24] for $\zeta \leq 1.6$ [Eqs. (b) and (B) in Tables $\Pi$ and $\amalg$. The dash-dotspace line is plotted using the fit formula of Itoh et al. [19] $(\zeta \leq 5.4)$. The dotted line in Fig. 66 shows the results of Ogata et al. 17, 23] ( $\zeta \lesssim 2$, Tables [1] and 【II) and the long-dashed line shows the PIMC calculations of Ogata [18] (also $\zeta \lesssim 2$, the same tables). We see that adding $h_{1}$ makes the reaction rate at $T \sim 0.1 T_{p}$ almost temperature independent, as it should be in the pycnonuclear regime.

It is remarkable that all cited results (except for Refs. [17, 23]) obtained at $T \gtrsim(0.1-0.2) T_{p}$ in different techniques and using various simplified assumptions (Sec. IIC) give actually almost one and the same reaction rate (almost the same curve in Fig. 6) reproduced by the mean-field WKB approach. It gives us confidence that this approach is really valid at $T \gtrsim(0.1-0.2) T_{p}$, and can now be considered as very reliable. It has been expected by many authors (e.g., Ref. [19]), and it is strictly confirmed by the Militzer-Pollock PIMC calculations 9].

\section{Pycnonuclear Militzer-Pollock data [group (iii)]}

Finally let us analyze six Militzer-Pollock data points which correspond to temperature-independent pycnonuclear burning [the data of group (iii) in Table IV. In Fig. 7 we plot the zero-temperature pycnonuclear carbon burning rate as a function of the density parameter $r_{s}$ defined by Eq. (3). For simplicity, in this figure we use a constant (energy independent) astrophysical factor $S(E=1 \mathrm{MeV})=3.2 \times 10^{16}$ barn $\mathrm{MeV}^{-1}$ for calculating the reaction rates. The six Militzer-Pollock reaction rates are shown by open dots.
We have compared these data with theoretical calculations of other authors. The shaded strip shows other theoretical predictions taking into account the uncertainties of various approximations. It is plotted using the expressions of Gasques et al. [10] who analyzed calculations of different authors. The strip is restricted by the minimum and maximum allowable reaction rates suggested in [10]. The solid line is the optimal theoretical reaction rate, which is the static-lattice model of Salpeter and Van Horn [8] for a bcc Coulomb crystal.

The consistency of the Militzer-Pollock data with other results is satisfactory. One should take in mind that the PIMC calculations in the pycnonuclear regime can be not too accurate because they require best computer resources. Moreover, the theoretical predictions of Ref. 10] refer to pycnonuclear reactions in Coulomb crystal while the actual state of ions in the PIMC runs is unknown (not reported in [9]). As pointed out by many authors (see, e.g., Refs. [32, 33, 34]), strong zero-point vibrations of ions at $r_{s} \gtrsim 90-160$ prevent their crystallization even at $T=0$. Therefore, three low- $r_{s}$ points of Militzer and Pollock in Fig. 7 should correspond to pycnonuclear burning in cold quantum liquid. However they do not deviate strongly from the predictions for Coulomb crystals.

Nevertheless, the agreement of the pycnonuclear Militzer-Pollock points with the best theoretical prediction of Salpeter and Van Horn [8] is not perfect. Filled dots in the inset in Fig. 7 display the ratio of the MilitzerPollock to the best Salpeter-Van Horn reaction rates; the errorbars are those reported in [9] (with the corrections mentioned in Sec. III). We see that the Militzer-Pollock rates are noticeably lower. The nature of this difference is unknown.

We expect that the Militzer-Pollock calculations in the pycnonuclear regime are not superior over other theoretical predictions at low $T$. More extended PIMC studies in the pycnonuclear regime would be helpful to reduce current theoretical uncertainties of the reaction rates.

\section{CONCLUSIONS}

We have analyzed the recent Path Integral Monte Carlo (PIMC) calculations by Militzer and Pollock [9] of contact probabilities of atomic nuclei participating in fusion reactions in dense matter. We have compared these calculations with other theoretical predictions. In particular, we have used a simple model based on WKB radial Coulomb tunneling of the reacting nuclei in the static mean-field plasma potential created by plasma ions. We have employed accurate Monte Carlo (MC) calculations of the mean-field plasma potential for a one-component strongly coupled plasma of ions and proposed a simple and accurate analytic fit to the plasma potential (Sec. IVA).

Our main conclusions are as follows:

1. We have found a very good agreement of the Militzer-Pollock PIMC results with the mean-field 
WKB calculations for $T \gtrsim(0.1-0.2) T_{p}$, i.e., in the thermonuclear regime, intermediate thermopycnonuclear regime and at highest temperatures of pycnonuclear burning (Table I). These results show good agreement with theoretical predictions of many authors (e.g., [16, 18, 19, 24]) and can be considered as well established.

2. There is a tentative slight disagreement of the PIMC and mean-field WKB results in the case of moderately strong ion coupling $\Gamma \lesssim 1$ but it cannot strongly affect the reaction rates.

3. We have obtained a very accurate fit (Sec. IV C) to the plasma screening enhancement factors calculated in the mean-field WKB approximation. The fit reliably describes the PIMC results in a wide temperature range $T \gtrsim(0.1-0.2) T_{p}$.

4. New studies of Coulomb tunneling problem in the pycnonuclear regime are needed to obtain accurate reaction rates in this regime.

The validity of the mean-field WKB method at $\Gamma \gtrsim 1$ and $T \gtrsim T_{p}$ could be expected (strong Coulomb coupling arranges quasi-order which may be well described by a radial mean-field without fluctuations). In contrast, there is little doubt that at $T \ll T_{p}$ the radial mean-field WKB picture is not true. At these low temperatures the reacting ions occupy quantum energy levels in their potential wells; they fuse along selected (anisotropic) closeapproach trajectories [8] which is definitely beyond the mean-field radial WKB method. Because the accuracy of PIMC calculations [9] decreases at low $T$, a nice agreement between the PIMC [9] and mean-field WKB approaches at $T \sim 0.1 T_{p}$, which we formally reached, may indicate insufficient accuracy of the low- $T$ PIMC results. New PIMC simulations would be most desirable to clarify this point.

\section{Acknowledgments}

We are grateful to B. Militzer, V. K. Nikulin, and A. Y. Potekhin for useful remarks. Work of AIC and DGY was partly supported by the Russian Foundation for Basic Research (grants 05-02-16245, 05-02-22003), by the Federal Agency for Science and Innovations (grant NSh 9879.2006.2), and by the Dynasty Foundation. Work of HED was performed under the auspices of the US Department of Energy by the Lawrence Livermore National Laboratory under contract number W-7405-ENG-48.
[1] D. D. Clayton, Principles of Stellar Evolution and Nucleosynthesis (University of Chicago Press, Chicago, 1983).

[2] P. Höflich, Nucl. Phys. A 777, 579 (2006).

[3] T. Strohmayer and L. Bildsten, in Compact Stellar XRay Sources, edited by W. H. G. Lewin, M. Van der Klis (Cambridge University Press, Cambridge, 2006), p. 113.

[4] A. Cumming, J. Macbeth, J. J. M. in 't Zand, and D. Page, Astrophys. J. 646, 429 (2006).

[5] S. Gupta, E. F. Brown, H. Schatz, P. Moeller, and K.-L. Kratz, Astrophys. J. 662, 1188 (2007)

[6] D. Page, U. Geppert, and F. Weber, Nucl. Phys. A777, 497 (2006).

[7] K. P. Levenfish and P. Haensel, Astrophys. Space Sci. 308, 457 (2007).

[8] E. E. Salpeter and H. M. Van Horn, Astrophys. J. 155, 183 (1969).

[9] B. Militzer, E. L. Pollock, Phys. Rev. B 71, 134303 (2005).

[10] L. R. Gasques, A. V. Afanasjev, E. F. Aguilera, M. Beard, L. C. Chamon, P. Ring, M. Wiescher, and D. G. Yakovlev, Phys. Rev. C 72, 025806 (2005).

[11] D. G. Yakovlev, L. R. Gasques, A. V. Afanasjev, M. Beard, and M. Wiescher, Phys. Rev. C 74, 035803 (2006).

[12] E. E. Salpeter, Aust. J. Phys. 7, 373 (1954).

[13] D. G. Yakovlev and D. A. Shalybkov, Soviet Sci. Rev. Sec. E 7, 313 (1989).

[14] H. E. DeWitt, H. C. Graboske, and M. S. Cooper, Astrophys. J. 181, 439 (1973).

[15] Y. Rosenfeld, Phys. Rev. E 53, 2000 (1996).

[16] B. Jancovici J. Stat. Phys. 17, 357 (1977).

[17] S. Ogata, H. Iyetomi, and S. Ichimaru, Astrophys. J. 372,
259 (1991).

[18] S. Ogata, Astrophys. J. 481, 883 (1997).

[19] N. Itoh, F. Kuwashima, and H. Munakata, Astrophys. J. 362, 620 (1990).

[20] A. Y. Potekhin and G. Chabrier, Phys. Rev. E 62, 8554 (2000).

[21] H. DeWitt and W. Slattery, Contrib. Plasma Phys. 43, 279 (2003).

[22] H. DeWitt and W. Slattery, Contrib. Plasma Phys. 39, 97 (1999).

[23] S. Ogata, S. Ichimaru, and H. M. Van Horn, Astrophys. J. 417, 265 (1993).

[24] A. Alastuey and B. Jancovici, Astrophys. J. 226, 1034 (1978).

[25] H. Kitamura, Astrophys. J. 539, 888 (2000).

[26] H. Kitamura and S. Ichimaru, Astrophys. J. 438, 300 (1995).

[27] C. L. Jiang, K. E. Rehm, B. B. Back, and R. V. F. Janssens, Phys. Rev. C 75015803 (2007).

[28] S. Ichimaru, Rev. Mod. Phys. 65, 255 (1993).

[29] J.-M. Caillol and D. Gilles, J. Phys. A 36, 6243 (2003).

[30] N. Itoh, N. Tomizawa, S. Wanajo, and S. Nozawa, Astrophys. J. 586, 1436 (2003).

[31] B. Widom, J. Chem. Phys. 39, 2808 (1963).

[32] D. M. Ceperley and B. J. Alder, Phys. Rev. Lett. 45, 566 (1980).

[33] G. Chabrier, Astrophys. J. 414, 695 (1993).

[34] M. D. Jones and D. M. Ceperley, Phys. Rev. Lett. 76, 4572 (1996). 